CARDIOLOGY

\title{
Mitral Valve Stenosis: Still a Clinical Challenge?
}

\author{
Andrew Xanthopoulos ${ }^{a}$ b Randall C. Starling ${ }^{b}$ Filippos Triposkiadis ${ }^{a}$ \\ ${ }^{a}$ Department of Cardiology, Larissa University General Hospital, Larissa, Greece; ${ }^{\text {b}}$ Department of Cardiovascular \\ Medicine, Heart and Vascular Institute, Kaufman Center for Heart Failure, Cleveland Clinic, Cleveland, OH, USA
}

The left atrium (LA) is a thin-walled structure located in the inflow path between the pulmonary veins and the left ventricle (LV) with important mechanical, neurohormonal, and regulatory actions [1]. The mechanical function of the LA consists of 3 phases: (a) the filling or reservoir phase during LV systole - when blood originating from the pulmonary veins is stored in the LA, (b) the conduit and passive emptying phase during early LV filling - when blood flows from the pulmonary veins through the LA to the LV (conduit) and at the same time blood stored in the LA during the reservoir phase flows into the LV due to existing LA-LV pressure gradients (passive emptying), and (c) the active emptying phase (atrial contraction) during late LV filling - when the LA contraction at LV end-diastole ejects blood into the LV and contributes to approximately $20-30 \%$ of total LV stroke volume in healthy subjects with sinus rhythm (SR). The contribution of the LA contraction to LV filling and LV stroke volume increases significantly when mitral valve stenosis (MVS) or LV dysfunction exist. Eventually LA remodeling (LA dilation plus LA hypocontractility) develops as a result of chronic LA mechanical overload, eventually often leading to atrial fibrillation (AF) with loss of atrial contraction, which is associated with reduced exercise capacity [1].

Rheumatic mitral valve stenosis (rMVS), the most common cause of MVS worldwide, leads to increased LA pressure (pressure overload), decreased forward flow to the LV, and eventually to pulmonary hypertension and heart failure symptoms [2]. Initially, patients with rMVS

\section{KARGER}

() 2018 S. Karger AG, Basel

E-Mail karger@karger.com

www.karger.com/crd in SR manage to compensate flow impediment from the LA to the LV with an increase in LA dimensions associated with augmented LA contraction (Frank-Starling mechanism), which maintains LA total emptying volume [3]. Eventually, LA remodeling develops resulting from tachycardia and the chronic pressure overload, which trigger several histological changes in the LA, including hypertrophy, necrosis, apoptosis, and fibrosis, and promoting the development of AF $[4,5]$. Thus, not surprisingly, inhibition of development or regression of existing LA remodeling (LA reverse remodeling), whatever the underlying cause, have been therapeutic targets with the use of drugs (e.g., neurohumoral inhibitors in heart failure), device treatment (cardiac resynchronization therapy in heart failure), and interventional/surgical management (mitral valve disease), albeit with variable success. Evidence deriving from a prospective study of 303 patients who underwent successful percutaneous mitral balloon commissurotomy (PMBC) and had been followed up for a mean of 10 years showed that LA volume decreased significantly immediately after PMBC and was sustained until 1 year after PMBC [6]. LA volume subsequently increased exceeding the pre-PMBC level by 8 years after PMBC. Multivariate analysis indicated that LA volume increase 10 years after $\mathrm{PMBC}$ was independently related to the post-PMBC mitral valve area, the echo score, the presence of $\mathrm{AF}$, and post-PMBC LA volume. Most importantly, the progressive increase in post-PMBC LA volume, which was observed even after successful 
percutaneous mitral valvuloplasty, was associated with a poor prognosis [6].

$\mathrm{PMBC}$ is a safe and effective treatment modality when applied in carefully selected patients with rMVS. In this regard, a useful tool is the echocardiographic Wilkins score, which derives from the anatomical characteristics of the mitral valve and includes 4 features; leaflet mobility, valvular thickening, valvular calcification, and subvalvular thickening [7]. Even though after successful PMBC most patients will maintain the preprocedural rhythm (patients with SR will remain on SR and those with AF will remain on $\mathrm{AF}$ ), there is no consensus regarding earlier implementation of $\mathrm{PMBC}$ with the purpose to prevent the development of permanent $\mathrm{AF}$, an independent predictor of major adverse cardiac events after percutaneous mitral valvuloplasty [8]. Performance of PMBC in asymptomatic patients with severe MVS (mitral valve area $\leq 1.5 \mathrm{~cm}^{2}$ ) and favorable valve morphology with new onset or paroxysmal AF has a class IIb recommendation in the American guidelines and IIa in the recent European guidelines for valvular heart disease $[9,10]$.

In this issue of Cardiology, Cho et al. [11] present the results of a retrospective study of 107 patients with rMVS who underwent successful PMBC and were followed up with echocardiography for at least 10 years. The authors observed that almost half of the patients who underwent PMBC were still on SR at the very long-term follow-up and that the maintenance of the SR correlated with the presence of preprocedural SR and LA diameter $<50 \mathrm{~mm}$. Two thirds and $90 \%$ of patients with preprocedural SR and AF, respectively, maintained the same rhythm at the very longterm follow-up. Furthermore, they observed that the group of patients who were on SR at the 10-year follow-up exhibited smaller LV and LA dimensions and higher LV ejection fraction than subjects who were in AF. Notably, the above echocardiographic findings were accompanied by higher event-free survival in the SR subgroup than in the AF subgroup. These findings are in accordance with those of previous reports $[8,12]$. The major limitations of this study are its retrospective nature and the fact that the choice for PMBC intervention was not only based upon "proper" valve-related parameters for percutaneous intervention but also on physician/patient preferences. In addition, litthe is known about the number of patients who might have undergone spontaneous, pharmaceutical, or electrical cardioversion during the follow-up time and eventually restored SR. However, the small number of patients on antiarrhythmic drugs after PMBC denotes that those who cardioverted and restored SR at follow-up were most likely a minority. Despite these limitations, this important paper by Cho et al. [11] provides further evidence supporting the detrimental effects of AF after PMBC. Although PMBC does not have an established antiarrhythmic action regarding incident AF despite postinterventional hemodynamic improvement, the results of their study favor an earlier intervention in asymptomatic patients with severe MVS who are at increased risk to develop permanent AF. The study also provides evidence that once AF is established in the setting of pathological LA changes, it will most likely remain unchanged even after relief of MVS. Resolving this issue necessitates a large prospective trial. However, such a trial is unlikely to be conducted in Western countries due to the low prevalence and incidence of rheumatic fever and MVS.

\section{References}

-1 Rossi A, Gheorghiade M, Triposkiadis F, Solomon SD, Pieske B, Butler J: Left atrium in heart failure with preserved ejection fraction: structure, function, and significance. Circ Heart Fail 2014;7:1042-1049.

$>2$ Harb SC, Griffin BP: Mitral valve disease: a comprehensive review. Curr Cardiol Rep 2017;19:73.

-3 Triposkiadis F, Wooley CF, Boudoulas H: Mitral stenosis: left atrial dynamics reflect altered passive and active emptying. Am Heart J 1990;120:124-132.

-4 Nattel S, Burstein B, Dobrev D: Atrial remodeling and atrial fibrillation: mechanisms and implications. Circ Arrhythm Electrophysiol 2008; 1:62-73.

$>5$ Triposkiadis F, Pieske B, Butler J, et al: Global left atrial failure in heart failure. Eur J Heart Fail 2016;18:1307-1320.
-6 Kim KH, Kim YJ, Shin DH, et al: Left atrial remodelling in patients with successful percutaneous mitral valvuloplasty: determinants and impact on long-term clinical outcome. Heart 2010;96:1050-1055.

7 Wilkins GT, Weyman AE, Abascal VM, Block PC, Palacios IF: Percutaneous balloon dilatation of the mitral valve: an analysis of echocardiographic variables related to outcome and the mechanism of dilatation. Br Heart J 1988; 60:299-308.

8 Tomai F, Gaspardone A, Versaci F, et al: Twenty year follow-up after successful percutaneous balloon mitral valvuloplasty in a large contemporary series of patients with mitral stenosis. Int J Cardiol 2014;177:881-885.

$\checkmark 9$ Nishimura RA, Otto CM, Bonow RO, et al: 2014 AHA/ACC guideline for the management of patients with valvular heart disease: a report of the American College of Cardiology/American Heart Association Task Force on Practice Guidelines. J Thorac Cardiovasc Surg 2014;148:e1-e132; erratum J Thorac Cardiovasc Surg 2014;64:1763.

10 Falk V, Baumgartner H, Bax JJ, et al: 2017 ESC/EACTS guidelines for the management of valvular heart disease. Eur J Cardiothorac Surg 2017;52:616-664.

-11 Cho I-J, Kim S-J, Han D, Kim D, Shim CY, Hong G-R, Chang H-J: Different characteristics, clinical outcomes, and left atrial reverse remodeling in patients with mitral stenosis maintaining sinus rhythm for at least 10 years after successful percutaneous mitral valvuloplasty. Cardiology 2018;140:14-20.

-12 Triposkiadis F, Trikas A, Tentolouris K, et al: Effect of atrial fibrillation on exercise capacity in mitral stenosis. Am J Cardiol 1995;76:282-286. 